\title{
Author Correction: The spread of steppe and Iranian-related ancestry in the islands of the western Mediterranean
}

Daniel M. Fernandes (D), Alissa Mittnik (D), Iñigo Olalde, losif Lazaridis, Olivia Cheronet, Nadin Rohland, Swapan Mallick, Rebecca Bernardos (D), Nasreen Broomandkhoshbacht Di, Jens Carlsson, Brendan J. Culleton, Matthew Ferry, Beatriz Gamarra, Martina Lari, Matthew Mah D, Megan Michel, Alessandra Modi D, Mario Novak, Jonas Oppenheimer, Kendra A. Sirak, Kristin Stewardson, Kirsten Mandl, Constanze Schattke, Kadir T. Özdoğan, Michaela Lucci, Gabriella Gasperetti, Francesca Candilio (D), Gianfranca Salis, Stefania Vai DD, Edgard Camarós (D), Carla Calò, Giulio Catalano, Marián Cueto (D), Vincenza Forgia (D), Marina Lozano DD, Elisabetta Marini, Margherita Micheletti, Roberto M. Miccichè (iD, Maria R. Palombo, Damià Ramis, Vittoria Schimmenti, Pau Sureda (D), Luís Teira, Maria Teschler-Nicola, Douglas J. Kennett, Carles Lalueza-Fox (D), Nick Patterson, Luca Sineo (iD, Alfredo Coppa, David Caramelli (iD, Ron Pinhasi (iD) and David Reich (ID)

Correction to: Nature Ecology \& Evolution https://doi.org/10.1038/s41559-020-1102-0, published online 24 February 2020.

In the version of this Article originally published, a sentence in the penultimate paragraph of the Discussion section should not have referred to the Balearic Islands. The sentence 'For example, all six individuals in our analysis dataset from the Balearic Islands and post-Bronze Age period are consistent with having no ancestry from earlier local groups.' should have read 'For example, all six individuals in our analysis dataset from the post-Bronze Age period are consistent with having no ancestry from earlier local groups.'

Additionally, in the Methods subsection 'qpWave and qpAdm', the word 'intersection' in the penultimate sentence should have been 'union'; the sentence 'We used the option allsnps: YES to specify that the $f_{4}$-statistics, which are the basis of qpWave and qpAdm analyses, should be computed using the intersection of SNPs with coverage in all four groups that contribute to each $f_{4}$-statistic.' should have read 'We used the option allsnps: YES to specify that the $f_{4}$-statistics, which are the basis of qpWave and qpAdm analyses, should be computed using the union of SNPs with coverage in all four groups that contribute to each $f_{4}$-statistic.'

These errors have now been corrected.

Published online: 15 April 2020

https://doi.org/10.1038/s41559-020-1197-3

(C) The Author(s), under exclusive licence to Springer Nature Limited 2020 